\title{
Efficiency of Minimized Circuits of a Heart Roller Pump on Systemic Inflammatory Response Syndrome and Multiorgan Effects in a Rat Model
}

\author{
Serdar Bayrak, ${ }^{1}$ Tugra Gencpinar, ${ }^{1}$ Gokmen Akkaya, ${ }^{2}$ Cagatay Bilen, ${ }^{2}$ Pınar Akokay, ${ }^{3}$ Nuran Aydereli, ${ }^{1}$ \\ Osman Yilmaz, ${ }^{4}$ Kıvanç Metin ${ }^{1}$ \\ ${ }^{1}$ Department of Cardiovascular Surgery, Dokuz Eylül University Faculty of Medicine, İzmir, Turkey; ${ }^{2}$ Department of Cardiovascular \\ Surgery, Ege University Faculty of Medicine, İzmir, Turkey; ${ }^{3}$ Medical Laboratory Technigues Programme, İzmir Kavram Vocational \\ School, İzmir, Turkey; 4Department of Multidisciplinary Laboratory Animal Science, Dokuz Eylül University Faculty of Medicine, \\ İzmir, Turkey
}

\section{ABSTRACT}

Background: The aim of this study is to compare the effects of tubing length on systemic inflammatory response syndrome and myocardial protection in a rat model of cardiopulmonary bypass (CPB) from a histological standpoint.

Methods: Twelve adult male Wistar Albino rats weighing $>180 \mathrm{~g}$ were randomly selected and divided into 2 groups. In 1 group, the pump lines were kept $1 \mathrm{~m}$ shorter than standard. The right jugular vein and tail artery were cannulated using a 16-gauge catheter. Animals received $500 \mathrm{IU} / \mathrm{kg}$ intravenous heparin. Cardiac index and rectal temperature were set at $2.4 \mathrm{~mL}$ and $36^{\circ} \mathrm{C}$, respectively. Total line volume was maintained at $8 \mathrm{~mL}$. A roller pump was adjusted to supply a blood flow of 6 to $28 \mathrm{~mL} / \mathrm{min}$ (mean $10 \mathrm{~mL} / \mathrm{min}$ ), similar to the typical cardiac output of rats. CPB duration was 15 minutes throughout the experiment. After sacrifice, tissue samples were collected from heart, liver, and kidney for histomorphologic examination.

Results: All histochemical and histomorphologic analyses, performed by 2 blinded researchers, revealed band loss in cardiomyocytes, mononuclear (MNL) cell infiltration, and impaired fibrillar organization in the standard-line group. Additionally in that group, sinusoidal dilatation in the liver, low-level congestion, focal necrosis, and periportal MNL infiltration were noted. In the shorter-line group, on the other hand, MNL cell infiltration, band loss in myofibrils, and cardiomyocyte degeneration were rarely observed. Higher liver congestion and lower MNL cell infiltration were observed in the shorter-line group. No significant differences were found in kidney samples.

Conclusion: In a shorter-line roller pump test model, less multiorgan damage and fewer systemic inflammatory responses were observed. It may be applicable to keep CPB lines as close to the table as possible, especially in pediatric cardiac surgery cases.

Received December 6, 2019; received in revised form February 9, 2020; accepted February 10, 2020.

Correspondence: Tugra Gencpinar, MD, Cardiovascular Surgery Department, Izmir, Turkey; 905054332289 (e-mail: tugra01@yahoo.com).

\section{INTRODUCTION}

Even with minimally invasive techniques, cardiopulmonary bypass $(\mathrm{CPB})$ is an essential component of cardiac surgery. Detection of myocardial damage caused by $\mathrm{CPB}$ or extracorporeal circulation is an important clinical problem.

Because of nonendothelialized pump lines, gas and particle embolisms, and flow alterations, CPB has a destructive effect on all tissues and organs, despite technical developments and increased experience [Shinozaki 2015; Peterss 2014]. During CPB, various organ systems can be adversely affected.
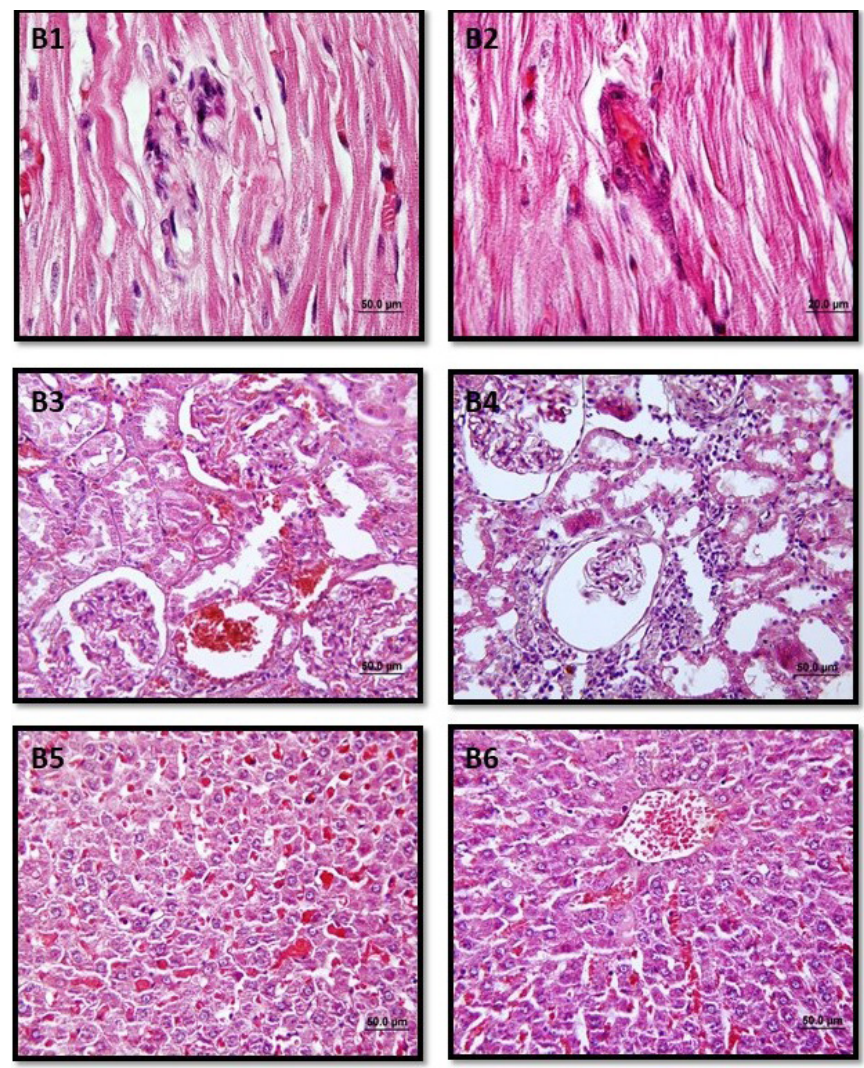

Figure 1. Evaluation of general histomorphology. B1 to B6, standard line. H\&E. 

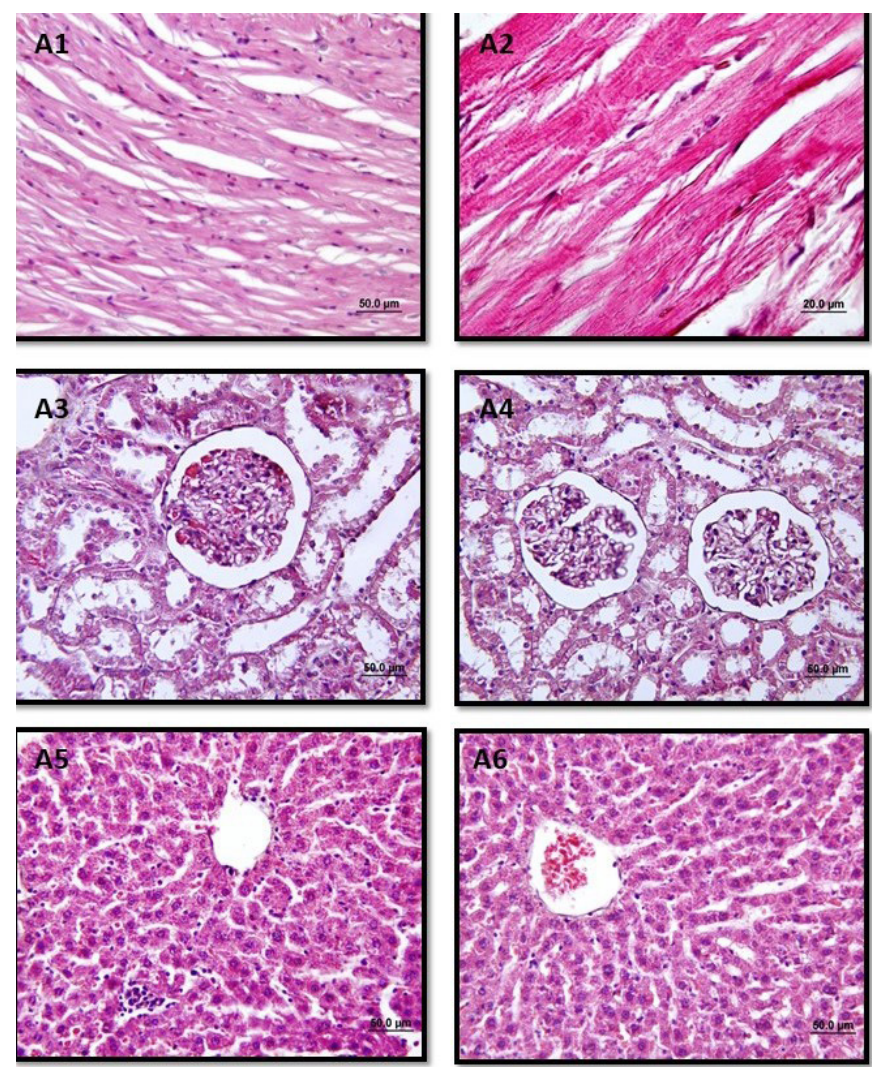

Figure 2. Evaluation of general histomorphology. A1 to A6, short line. H\&E.

Systemic inflammatory response syndrome (SIRS) might be triggered during $\mathrm{CPB}$. Postperfusion syndrome may cause pulmonary and renal dysfunction, neurological and gastrointestinal injury, coagulation cascade disorders, interstitial fluid increase, and suspicious infections [Engels 2014; Zhang 2015; Zhou 2015; Fujii 2015; Koster 2009]. Nonphysiologic flow and resistance changes applied during $\mathrm{CPB}$ affect organ perfusion. Therefore, hypoperfusion and ischemia might occur first in the central nervous system, then in the kidneys and other vital organs. Formed blood elements, platelets, coagulation factors, and related blood proteins are damaged because of heparinization, neutralization, and blood circulation in a nonendothelialized environment. As a result of that damage, severe complications such as bleeding, SIRS, hemolysis, and even intravascular coagulation syndrome might emerge [Shinozaki 2015; Peterss 2014; Kim 2015; Wang 2015; Engels 2014].

Zhang [2015] studied brain protection in the rat model of $\mathrm{CPB}$ and reported that the perioperative period of $\mathrm{CPB}$ correlated with cerebral damage. Zhou [2015] emphasized that immune cascades are triggered by surgical equipment and CPB lines, a situation that primarily leads to receptormediated brain damage, which can be prevented by anesthetic agents such as neuroprotective sevoflurane. Fujii [2015, 2016] reported that the large surface area that blood contacts during CPB initiates strong inflammatory responses
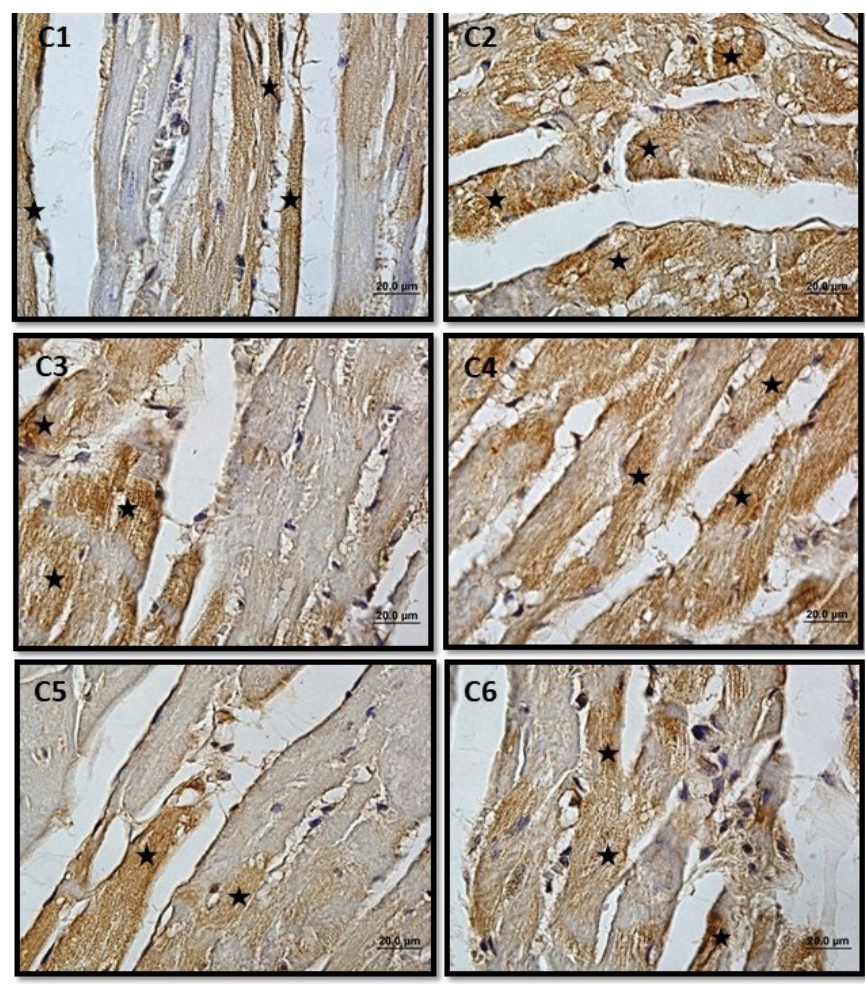

Figure 3. Immunohistochemical examination of the rat heart tissue with anti-NOS. Black star indicates iNOS immunopositive cells. Anti-iNOS immunopositive cardiomyocytes were higher in the standard-length roller pump model than in the shorter-line model. Bar: $20 \mu \mathrm{m}$, original magnification $40 \times$.

by triggering hormonal and humoral autacoids in the form of chain reactions. They also noted that decreased surface area might reduce vital organ damage by reducing inflammation, and that studies showing organ damage are needed. In their study, serum cytokine levels and biochemical markers were evaluated.

Shinozaki [2015] declared that, at the end of the procedure, they encountered anemia in an experimental animal model in which they continuously used hemofiltration under CPB and resuscitation. Petters [2014] reported that the effects of hemodilution, inflammatory response, low blood pressure, and reperfusion injury that might result from $\mathrm{CPB}$ are not well known, and that further experimental studies are needed. Wang [2015] reported that mitochondrial free oxygen radicals are elevated in the ischemia-reperfusion period, which in turn caused cerebral injury and neurocognitive impairment in a rat model of CPB. It has also been noted that the condition correlated with increases in CPB exposure. As emphasized in that study, efforts to minimize the effects of CPB are crucial. Schnoering [2013a,b] reported that reduced priming volume in experimental animal studies with a minimal heart-lung machine does not reduce the inflammatory response.

In a randomized controlled study conducted by Rahman [2005], the use of a mini-bypass unit resulted in marked suppression of coagulation and activation of the fibrinolytic 
Hemodynamic Parameters and Blood Gas Sample Analyses

\begin{tabular}{lccc}
\hline Characteristic & $\begin{array}{c}\text { Baseline } \\
(\mathrm{n}=12)\end{array}$ & $\begin{array}{c}\text { Short line } \\
(\mathrm{n}=6)\end{array}$ & $\begin{array}{c}\text { Standard } \\
\text { line }(\mathrm{n}=6)\end{array}$ \\
\hline Weight $(\mathrm{g})$ & $310 \pm 8$ & $313 \pm 3$ & $315 \pm 6$ \\
Mean arterial pressure $(\mathrm{mmHg})$ & $70 \pm 8$ & $71 \pm 6$ & $72 \pm 4$ \\
Heart rate (per min) & $313 \pm 2$ & $318 \pm 2$ & $322 \pm 3$ \\
Flow (mL/min) & & $10 \pm 3$ & $10 \pm 1$ \\
Temperature $\left({ }^{\circ} \mathrm{C}\right)$ & $36.5 \pm 0.3$ & $36.3 \pm 0.5$ & $36.0 \pm 0.1$ \\
$\mathrm{pH}$ & $7.33 \pm 0.05$ & $7.32 \pm 0.08$ & $7.30 \pm 0.04$ \\
Partial pressure $\mathrm{CO}_{2}(\mathrm{mmHg})$ & $47 \pm 2$ & $47 \pm 5$ & $48 \pm 3$ \\
Partial pressure $\mathrm{O}_{2}(\mathrm{mmHg})$ & $103 \pm 6$ & $101 \pm 6$ & $98 \pm 2$ \\
$\mathrm{~K}(\mathrm{mEq} / \mathrm{L})$ & $4.3 \pm 0.3$ & $4.5 \pm 0.3$ & $4.6 \pm 0.2$ \\
Hematocrit $(\%)$ & $43 \pm 3$ & $42 \pm 4$ & $42 \pm 2$
\end{tabular}

cascade, and no reduction in blood loss was observed compared with conventional CPB. In that system, neither a cardiotomy reservoir nor a pericardial aspirator was used to prevent blood-air contact. By reducing the priming volume from 1700 to $500 \mathrm{~mL}$ and the surface area from 12 to $1.4 \mathrm{~m}^{2}$, contact with the nonendothelialized surfaces of the $\mathrm{CPB}$ machine was decreased, and prothrombotic and proinflammatory responses were attenuated. The authors reported that complex pump studies showing clinical benefit are required [Rahman 2005].

To evaluate the pathophysiology of this nonphysiological condition, experimental animal models are still necessary. Additionally, patient-device interactions are not fully known. The purpose of this study is to compare the effects of shortened tubing length on systemic inflammatory response syndrome and myocardial protection in an experimental rat model.

\section{METHODS}

Our randomized controlled experimental study was carried out with the approval of Dokuz Eylul University Multidiscipline Laboratory Animal Experiments local ethics committee on December 15, 2015 (protocol number 54/2015). All procedures were carried out in accordance with the National Institutes of Health Animal Care Principles. Adult Wistar (400- to 450-g) albino male rats were used. The rat model is preferred owing to the high reliability of the findings and reproducibility of the experiments, and the animals are easily obtained from laboratory animal research centers. Moreover, the rat model is documented in the literature as an appropriate model for isolated CBP studies [Peterss 2014]. The use of male Wistar albino rats in our experimental study was decided after negotiating with the Histology-Morphology department. Twelve animals were randomly divided into 2 groups. The rats were obtained from and housed in Dokuz Eylul University laboratory animal research center under standard conditions with a 12 -h light-dark cycle and $55 \%$ to $60 \%$ humidity, and all experimental procedures of the present study were also carried out in that center. Animals were fed with standard rat diet ad libitum. For protection against infection, antibiotic therapy was applied by preoperative administration of cefazolin at a dose of $50 \mathrm{mg} / \mathrm{kg}$. In addition, the skin was shaved and cleaned with $10 \%$ povidone-iodine solution to provide better surgical field visibility. The rectal temperature was kept at an average of $36.5^{\circ} \mathrm{C}$. The animals were heated with radiant heaters during the experiment.

\section{Line Length Calculations and Tubing Sets}

In the perfusion model with constant pressure, the pressure is kept constant so that when perfusion flow is measured, this value can be substituted for resistance, and the changes in the flow give direct information about the radius variations of the vessel. In the perfusion model with constant flow, when perfusion pressure is measured while keeping the flow constant, the measured values provide information about the changes of pressure and vessel radius.

In the perfusion model with constant pressure, a measurement achieved with the following formula,

resistance $=$ pressure $(\mathrm{mmHg}) \div$ current $(\mathrm{mL} / \mathrm{min})=1 \div$ vessel radius $\times 4$,

can replace the resistance and changes in flow directly from the changes in the radius of the vessel. On the other hand, if the perfusion pressure is measured with constant flow, the measurements give information about pressure changes and vessel radius changes.

Thus the following tubing sets were used: $1-\mathrm{m}, 0.25$-inch inner diameter (ID) $=32 \mathrm{~mL} ; 1-\mathrm{m}, 3 / 16$-inch ID $=18 \mathrm{~mL}$; and 1 - $\mathrm{m}, 0.125$-inch ID $=8 \mathrm{~mL}$. To reduce prime fluid, also used were an oxygenator (filter integrated system) or arterial filter with 1 tubing set (1 shortened and shrunk by 2 diameters).

\section{Experimental Groups}

Dokuz Eylul University Multidiscipline Laboratory Animal Experiments Local Ethics Committee approved the experiments. In the first group $(n=6)$, the line was shortened by 1 meter. Priming volume was kept to a minimum. The same procedure was applied to the second group $(n=6)$ with standard line length. Rats were sacrificed with $150 \mathrm{mg} /$ $\mathrm{kg}$ pentothal at minute $15 \mathrm{of} \mathrm{CPB}$ as an appropriate model for isolated CBP studies in the literature [Peterss 2014].

\section{Surgical Procedures}

All subjects $(\mathrm{n}=12)$ were anesthetized with intramuscular injection of $50 \mathrm{mg} / \mathrm{kg}$ ketamine hydrochloride (Ketalar, Pfizer, New York) and $5 \mathrm{mg} / \mathrm{kg}$ xylazine hydrochloride (Xylazine, Bayer Chemistry, Whippany, NJ). Once corneal reflex was lost, animals were fixed to the operation table in the supine position. Spontaneous respiration was maintained at a rate of $3 \mathrm{~L} / \mathrm{min}$ with an oxygen mask. Prophylactic antibiotic therapy was $50 \mathrm{mg} / \mathrm{kg}$ intramuscular cefazolin sodium (Bilim Pharmaceuticals, İstanbul, Turkey). A surgical loupe (3.5x, Designs for Vision, Bohemia, NY) was used during surgery and dissection. The right jugular vein and the tail artery were cannulated with a 16-gauge catheter. 
For the venous line, a 16-gauge intravenous catheter was placed into the right atrium through the vena jugularis externa after dissecting the neck. The tails of the rats were cleaned with $10 \%$ povidone-iodine (Poviiodeks, Kim Pharmaceuticals, Philadelphia), and the tail artery was cannulated with a 16-gauge catheter (Biçakçılar Company, Istanbul, Turkey) for the arterial line. $500 \mathrm{IU} / \mathrm{kg}$ heparin sodium was administered intravenously. Venous blood was pumped with a roller pump (Masterflex, Cole-Palmer, Vernon Hills, IL) through a silicone tube system (polyvinyl chloride) of ID 1.6 $\mathrm{mm}$, and the blood was returned to the subject by the arterial line. In the standard-line group, $14 \mathrm{~mL}$ of $0.040 \mathrm{~m}$ blood contact surface area, and in the short-line group $7 \mathrm{ml}(50 \%)$ surface area, was reduced by half to $0.020 \mathrm{~m}(50 \%)$. During the experiment, the cardiac index was maintained at $2.4 \mathrm{~mL}$. The total line length was kept to $2 \mathrm{~m}$ for the standard line, and the roller pump was adjusted to have a blood flow of 6 to $28 \mathrm{~mL} /$ min (mean $10 \mathrm{~mL} / \mathrm{min}$ ), similar to typical cardiac output for rats. Arterial blood samples of $0.2 \mathrm{~mL}$ were collected at the beginning and end of the CPB to obtain basal and end arterial blood gas levels (Stat profile pHOx Blood Analysis System, Novabio, Waltham, MA) (see Table). The initial hemoglobin value was noted to be $>8 \mathrm{mg} / \mathrm{dL}$ as indicated in the literature [Fujii 2015] ( $\mathrm{Hb}<7 \mathrm{~g} / \mathrm{dl}$ was determined to be an exclusion criterion). No blood transfusion was required. No rat was excluded from the study. The rats were hemodynamically stable with an arterial blood pressure of $70 \mathrm{mmHg}$, heart rate of $322 \pm 3 / \mathrm{min}$ (Petaş, KMA-250 monitor, Dilek Medical, Istanbul, Turkey), and $\mathrm{Hb}$ of $10 \mathrm{~g} / \mathrm{dL}$ during the procedure. None of the rats died during the model.

All rats were sacrificed 15 minutes postoperatively, and isolated rat hearts and other vital tissues (kidney and liver) were collected. Damage to myocardial tissue and systemic inflammatory response syndrome were evaluated in the histology and embryology laboratory. Tissue sections were examined under light microscope, and digital images were analyzed for tissue damage and SIRS. Pre- and postoperative routine biochemistry and hematology values were used to assess hemolysis and systemic response.

\section{Histomorphologic Evaluation}

Light Microscopy. Rat tissue samples were removed, fixed in $10 \%$ neutral buffered formalin, dehydrated in a graded series of isopropyl alcohol (60\% to $100 \%$ ) and xylol, and embedded in paraffin, and 5- $\mu \mathrm{m}$ sections were sliced with arotary microtome (RM 2255, Leica Instruments, Nussloch, Germany) on poly-L-lysine-coated slides. Sections stained with hematoxylin and eosin (H\&E) (Surgipath, Cambridgeshire, UK) were used to evaluate general histomorphology (Figures 1 and 2). Images were obtained from the selected areas and analyzed with a computer-assisted image analyzer system consisting of a microscope (BX-51, Olympus, Tokyo, Japan) equipped with a high-resolution video camera (DP70, Olympus). Measurements were made using UTHSCA Image Tool (version 3.0, University of Texas Health Science Center, San Antonio, TX).

Immunohistochemistry. For immunohistochemical staining, sections were deparaffinized and rehydrated in a decreasing alcohol series. Their surroundings were limited with a Dako-Pen without damaging the sections. The sections were kept in trypsin at $37^{\circ} \mathrm{C}$ for 15 minutes, and $3 \%$ hydrogen peroxide was applied for 10 minutes to inhibit endogenous peroxidase. The cross sections were washed with phosphatebuffered saline and incubated with blocking solution at room temperature for 20 minutes. iNOS primary antibody (bs0156R, Bioss, Woburn, MA) was applied to the cross sections and left at $4^{\circ} \mathrm{C}$ overnight. The cross sections were first incubated with a biotinylated secondary antibody and then with avidin-biotin complex (TP 060-HL, Thermo Fisher Scientific, Waltham, MA) for 30 minutes. Diaminobenzidine was used to make the reaction visible. Ground staining was made with Mayer's hematoxylin, and after the process of dehydration and xylol series, they were closed with Entellan.

Image Analysis. A computerized video camera-based image analysis system (UTHSCA Image Tool) was used. All available sections ( $\geq 3$ sections per vessel) were analyzed; only sections with obvious technical artifacts related to the staining procedure were excluded. After the staining process was completed, sections were examined with a light microscope (BX-50, Olympus), and images were transferred to a computer using a high-resolution camera (DP-70, Olympus). All sections were digitally photographed (Figure 3).

\section{Data Analysis and Statistics}

Correlations between nonparametric variables and significance were assessed using Fisher's exact test. A $P$ value $<.05$ was considered significant. Statistical Package for Social Sciences (SPSS, Chicago, IL) 15 and Excel (Microsoft, Redmond, WA) were used for all calculations and statistical analysis.

\section{RESULTS}

All histochemical and immunohistochemical analyses (Figures 1 and 2) were performed by 2 blinded researchers. Histochemical examinations revealed band loss in cardiomyocytes, mononuclear (MNL) cell infiltration, and impaired fibrillar organization in the standard-line group, as well as sinusoidal dilatations in the liver, a low level of congestion, and focal necrosis. In the shorter-line group, on the other hand, MNL cell infiltration, band loss in myofibrils, and cardiomyocyte degeneration were rarely observed. In addition, higher liver congestion and lower MNL cell infiltration were observed in the shorter-line group. No significant difference was found for kidney samples. Despite the $50 \%$ decrease in line length, there was no difference in hematocrit levels. The explanation for this phenomenon is that blood transfusion was not required. The rats were hemodynamically stable, with acceptable arterial blood pressure and hemoglobin levels during the procedure.

\section{DISCUSSION}

In the present study, the effects of tubing length shortening on SIRS and myocardial protection in an experimental rat 
model were examined. This experimental study was planned to investigate the histopathologic changes in myocardium and visceral organs in relation to the length of the roller pump lines. In the shorter-line roller pump model, less multiorgan damage and fewer systemic inflammatory responses were observed. The idea behind defining SIRS was to pinpoint a clinical response to a nonspecific insult of either multiple organ dysfunction syndrome (MODS) or the effects of CPB origin. Vital organs were damaged less as a positive effect of reduced nonendothelialized contact area.

The length of pump lines plays a role in myocardial injury as a source of extra volume [Koster 2009; Redlin 2011]. Ischemia leads to rapid metabolic and ultrastructural impairment in the myocardium [Engels 2014; Zhang 2015; Zhou 2015; Fujii 2015]. The high-energy phosphate deposits quickly dissipate. In the case of normothermic ischemia, irreversible myocardial damage occurs within 30 to 40 minutes [Engels 2014; Zhang 2015; Zhou 2015; Fujii 2015; Koster 2009]. In addition to structural changes due to ischemia during surgery, cytotoxic free oxygen radicals are formed during reperfusion [Wang 2015; Engels 2014; Zhang 2015; Zhou 2015]. Increases in cytokines, interleukins, and tumor necrosis factors have also been reported [Engels 2014; Zhang 2015; Zhou 2015; Fujii 2015]. Blood-associated endotoxemia and hemolysis-related blood loss may occur during $\mathrm{CPB}$ [Fujii 2015, 2016; Koster 2009; Redlin 2011; Shen 2011; Mackensen 2001; Schnoering 2013]. Hydroxyl radicals also effect the progression of inflammation in prolonged $\mathrm{CPB}$ [Zhou 2015; Fujii 2015]. Ischemia results in disrupted myocardial cell structure. The protection of myocardium against ischemic damage should begin in the preoperative period.

These days, myocardial damage reduction studies are conducted using different techniques by professional teams according to the characteristics of the patients and the surgical technique to be applied; yet there is no standard application in CPB. The use of different methods can be tailored to the experience of the team. Myocardial injury can be attenuated with priming volume reduction as a result of shortened pump lines. In neonatal cases, the use of bloodless priming for CPB has been reported [Koster 2009; Redlin 2011].

In cardiovascular surgery, studies on myocardial protection methods and effects during CPB are limited, and the experimental or clinical results of different applications have not been fully demonstrated. With technological improvements, the use of short-line and minimal extracorporeal circulation will become increasingly popular [Shinozaki 2015; Peterss 2014; Kim 2015; Wang 2015; Engels 2014; Zhang 2015; Zhou 2015]. Additionally, this technology would enable less activation of the coagulation cascade and blood transfusion, and so will be routinely used [Fujii 2015, 2016; Koster 2009; Redlin 2011; Shen 2011; Mackensen 2001]. Shortening of pump lines in CPB has great importance in preventing myocardial infarction, heart failure, severe arrhythmias, and prolonged intensive care stay, as well as hospitalization-related clinical conditions such as pneumonia, sepsis, or gastrointestinal system hemorrhages, by reducing SIRS [Schnoering 2013; Walker 2003; Immer 2005; Engels 2014; Rahman 2005; Modine 2006].
Ganushchak [2016] emphasized negative outcomes despite the benefits of minimal CPB systems. Vacuum systems with kinetic support can cause severe fluctuation by pressurizing the venous lines, and gas microembolisms might occur. They recommended a versatile perfusion system (VSM) with minor modifications in conventional CPB circuits, taking these potentially harmful side effects into consideration [Ganushchak 2016]. Anastasiadis [2013] reported at least 1,59 lower cost for each minimized CPB case. Our rat model is a kind of VSM model. We also established a shortline roller pump animal model. With its help, new studies comparing other parameters can be designed in the future. We tried to explain the effects of small-diameter short-line $\mathrm{CPB}$ surgery in routine congenital cardiac surgery in an experimental aspect. If a centrifugal pump is used instead of a roller pump, we observed in our clinical studies that hemolysis findings are different [Silistreli 2012].

Immunocytochemical staining of heart, liver, and kidney cells revealed different results in our experiment. Renal and hepatic cell results were similar, whereas cardiac cellular iNOS results were different in both groups. Longer pump lines resulted in higher anti-iNOS immunopositive staining of cardiomyocytes $(P=.026)$. This finding proves the effect of line length on a cellular basis (Figure 3).

Furthermore, histomorphologic evaluation of the effect of foreign surface-volume balance on the CPB by experimental pump model may be beneficial for heart surgery. With this rat model, pump line effects can be examined in a reproducible and easily accessible manner, reducing both cost and need for equipment. Few publications in the literature cover the effects of the length of in vivo CPB pump lines and the contact surface. In addition, the damage mechanisms of this harmful effect have not been fully defined.

\section{Limitations}

The primary limitation of our work is that data at the molecular level are limited. We plan to conduct future studies with a higher budget to correlate immunohistochemical changes with oxidative stress markers. Different priming fluids or oxygenators were not used, to avoid changing dependent variables. Under spontaneous breathing, roller pump line lengths were compared. Further studies are needed to clarify the effects of shorter lines during CPB.

\section{Conclusion}

Less multiorgan damage and fewer systemic inflammatory responses were observed in the shorter-line roller pump experiment model. In cardiac surgeries, CPB lines should be kept as close as possible to the table, especially for infants.

\section{REFERENCES}

Anastasiadis K, Fragoulakis V, Antonitsis P, et al. Coronary artery bypass grafting with minimal versus conventional extracorporeal circulation; an economic analysis. Int J Cardiol 2013;168:5336-5343.

Engels M, Bilgic E, Pinto A, et al. A cardiopulmonary bypass with deep hypothermic circulatory arrest rat model for the investigation of the 
systemic inflammation response and induced organ damage. J Inflamm (Lond) 2014;11:26.

Engels M, Bilgic E, Pinto A, et al. A cardiopulmonary bypass with deep hypothermic circulatory arrest rat model for the investigation of the systemic inflammation response and induced organ damage. J Inflamm 2014;11:26.

Fujii Y, Shirai M, Inamori S, et al. A novel small animal extracorporeal circulation model for studying pathophysiology of cardiopulmonary bypass. J Artif Organs 2015;18:35-39.

Fujii Y, Shirai M, Takewa Y et al. Cardiopulmonary bypass with lowversus high-priming volume: Comparison of infammatory responses in a rat model. ASAIO J 2016;62:289-290.

Ganushchak Y, Körver E, Yamamoto Y, et al. Versatile minimized system: A step towards safe perfusion. Perfusion 2016;31:295-299.

Immer FF, Pirovino C, Gygax E, et al. Minimal versus conventional cardiopulmonary bypass: Assessment of intraoperative myocardial damage in coronary bypass surgery. Eur J Cardiothorac Surg 2005;28:701-704.

Kim J, Lampe JW, Yin T, et al. Phospholipid alterations in the brain and heart in a rat model of asphyxia-induced cardiac arrest and cardiopulmonary bypass resuscitation. Mol Cell Biochem 2015;408:273-281.

Koster A. A new miniaturised cardiopulmonary bypass circuit reduces transfusion requirements during neonatal surgery. Initial experiences in 13 consecutive patients. J Thorac Cardiovasc Surg 2009;137:1565-1568.

Mackensen GB, Sato Y, Nellgard B, et al. Cardiopulmonary bypass induces neurologic and neurocognitive dysfunction in the rat. Anesthesiol 2001;95:1485-1491.

Modine T, Azzaoui R, Fayad G, et al. A recovery model of minimally invasive cardiopulmonary bypass in the rat. Perfusion 2006;21:87-92.

Peterss S, Guenther S, Kellermann K, et al. An experimental model of myocardial infarction and controlled reperfusion using a miniaturized cardiopulmonary bypass in rats. Interact Cardiovasc Thorac Surg
2014;19:561-566.

Rahman A, Martens S, Risteski P, et al. The use of minimized extracorporeal circulation system has a beneficial effect on hemostasis: A randomized clinical study. Heart Surg Forum 2005;9:1110.

Redlin M. Minimizing intraoperative hemodilution by use of a very low priming volume cardiopulmonary bypass neonates with transposition of the great arteries. J Thorac Cardiovasc Surg 2011;142:875-881.

Schnoering H, Arens J, Detering SM, et al. Development of a rabbit animal model for miniaturized heart-lung machines. ASAIO J 2013;59:152-156.

Schnoering H, Arens J, Detering SM, et al. Expression of inflammation in myocardial tissue of rabbits: Comparison of two miniaturized heartlung machines. Artif Organs 2013;37:541-548.

Shen L, Wang J, Liu K, et al. Hydrogen-rich saline is cerebroprotective in a rat model of deep hypothermic circulatory arrest. Neurochem Res 2011;36:1501-1511.

Shinozaki K, Lampe JW, Wang CH, et al. Developing dual hemofiltration plus cardiopulmonary bypass in rodents. J Surg Res 2015;195:196-203.

Silistreli E, Ugurlu B, Catalyurek H, et al. The effects of pulsatile flow characteristics on hemolysis, transfusion requirement, and hemostasis in open heart surgeries. Turk Gogus Kalp Dama 2012;20:217-222.

Walker G, Liddell M, Davis C: Extracorporeal life support: State of the art. Paediatr Respir Rev 2003;4:147-152.

Wang Y, Gu T, Shi E, et al. Inhibition of microRNA-29c protects the brain in a rat model of prolonged hypothermic circulatory arrest. J Thorac Cardiovasc Surg 2015;150:675-684.

Zhang K, Li M, Peng XC, et al. The protective effects of sufentanil pretreatment on rat brains under the state of cardiopulmonary bypass. Iran J Pharm Res 2015;14:559-566.

Zhou J, Zhou N, Wu XN, et al. Role of the Toll-like receptor 3 signaling pathway in the neuroprotective effect of sevoflurane pre-conditioning during cardiopulmonary bypass in rats. Mol Med Rep 2015;12:7859-7868. 